Pacific Journal of Mathematics

THE 2-LENGTH OF A FINITE SOLVABLE GROUP 


\title{
THE 2-LENGTH OF A FINITE SOLVABLE GROUP
}

\section{Fletcher Gross}

\begin{abstract}
One measure of the structure of a finite solvable group $G$ is its $p$-length $l_{p}(G)$. A problem connected with this measure is to obtain an upper bound for $l_{p}(G)$ in terms of $e_{p}(G)$, which is a numerical invariant of the Sylow $p$-subgroups of $\mathbf{G}$. This problem has been solved but the best-possible result is not known for $p=2$. The main result of this paper is that $l_{2}(G) \leqq$ $2 e_{2}(G)-1$, which is an improvement on earlier results. A secondary objective of this paper is to investigate finite solvable groups in which the Sylow 2-group is of exponent 4 . In particular it is proved that if $G$ is a finite group of exponent 12 , then the 2-length is at most 2 .
\end{abstract}

Introduction and discussion of results. The object of this paper is to obtain bounds for the 2-length of a finite solvable group. Following Hall and Higman [4], we call a finite group $G$ p-solvable if it possesses a normal series such that each factor group is either a $p$-group or a $p^{\prime}$-group. The $p$-length, $l_{p}(G)$, of such a group is the smallest number of $p$-groups which can occur as factor groups in such a normal series. $e_{p}(G)$ is defined to be the smallest $n$ such that $x^{p n}=1$ for all $x$ belonging to a Sylow $p$-subgroup of $G$.

For an odd prime $p$, it is proved in [4] that $l_{p}(G) \leqq e_{p}(G)$ if $p$ is not a Fermat prime and $l_{p}(G) \leqq 2 e_{p}(G)$ if $p$ is a Fermat prime. Furthermore these results are best-possible. A.H. M. Hoare [6] then proved that in a 2-solvable group $G, l_{2}(G) \leqq 3 e_{2}(G)-2$ provided that $l_{2}(G) \geqq 1$. The primary purpose of this paper is to prove the following improvement:

THEOREM A. If $G$ is a finite solvable group and $l_{2}(G) \geqq 1$, then $l_{2}(G) \leqq 2 e_{2}(G)-1$.

Feit and Thompson [1] have proved that solvability and 2-solvability are equivalent notions for finite groups. Thus no loss of generality is involved in requiring $G$ to be solvable in the theorem.

Theorem A will be shown to be an easy consequence of the following theorem about linear groups:

THEOREM B. Let $G$ be a finite solvable linear group over a field

Received July 13, 1964. This work, supported in part by a National Science Foundation Cooperative Fellowship, is based upon my doctoral dissertation at the California Institute of Technology. I wish to express my heartfelt appreciation to my advisor Marshall Hall, Jr. for his encouragement and helpful suggestions. 
$F$ of characteristic 2 and assume $G$ has no nontrivial normal 2subgroup. Then if $N$ is the largest normal 2'-subgroup of $G$ and if $g$ is an exceptional element of order $2^{m}$ in $G$, it follows that $g^{2^{m-1}}$ is in the largest normal 2-subgroup of $G / N$.

Here, following [4], an element $x$ of order $p^{n}$ in a linear group over a field of characteristic $p$ is said to be exceptional if $(x-1)^{p n_{-1}}=0$.

Whether or not Theorem A represents a best-possible result is not known, but it seems likely that further improvements can be made. Indeed, the author knows of no group whose 2-length exceeds its 2-exponent. In the special case of finite solvable groups satisfying $e_{2}(G)=2$, i.e., solvable groups whose Sylow 2-subgroups are of exponent 4, I think it likely that $l_{2}(G) \leqq 2$ instead of the bound $l_{2}(G) \leqq 3$ furnished by Theorem A.

In $\S 4$ of this paper, groups satisfying $e_{2}(G)=2$ are studied in more detail. A sufficient condition for $l_{2}(G) \leqq 2$ in this special case is established, and, as an application, we prove that $l_{2}(G) \leqq e_{2}(G)$ if $G$ is a finite group of exponent 12 .

2. Proof of Theorem A from Theorem B. For the rest of this paper we adopt the convention that all groups referred to are assumed finite, and, if $G$ is such a group, then $|G|$ denotes its order. If $H$ is a normal subgroup of $G$, we write $H \triangleleft G$.

We now recall the definition of the upper 2-series of the solvable group $G$ :

$$
1=P_{0} \leqq N_{0}<P_{1}<N_{1}<\cdots<P_{l} \leqq N_{l}=G .
$$

Here $N_{k} / P_{k}$ is defined to be the greatest normal 2 'subgroup of $G / P_{k}$ and $P_{k+1} / N_{k}$ the greatest normal 2-subgroup of $G / N_{k}$. The least integer $l$ such that $N_{l}=G$ is the 2-length $l_{2}(G)$. (If there is no danger of confusion we write simply $l_{2}$.)

It is proved in [4] that the automorphisms of $P_{1} / F$, where $F / N_{0}$ is the Frattini subgroup of $P_{1} / N_{0}$, induced by $G$ represent $G / P_{1}$ faithfully. Thus $G / P_{1}$ is faithfully represented as a linear group operating on $P_{1} / F$ $\left(P_{1} / F\right.$ is an elementary abelian 2 -group and so is considered as a vector space over the field with 2 elements).

Now if $l_{2}(G)=1$, the conclusion of $A$ is trivial. Also the $p$-length group is at most equal to the class of a Sylow $p$-subgroup [4, Theorem 1.2.6]. An immediate consequence of this is that if $G$ is solvable and $e_{2}(G)=1$, then $l_{2}(G)=1$. Thus $l_{2}=2$ implies that $e_{2} \geqq 2$ so the result again follows. Now if $l_{2}>2$, then $l_{2}\left(G / P_{2}\right)=l_{2}(G)-2 \geqq 1$ so that Theorem $\mathrm{A}$ would follow by induction on $l_{2}$ if we could prove that 
$e_{2}\left(G / P_{2}\right) \leqq e_{2}(G)-1$.

Now suppose $g$ is an element of maximal order $2^{m}$ in a Sylow 2-Sylow subgroup of $G / P_{1}$. If $g$ is not exceptional, then [4, Lemma 3.1.2] we have $e_{2}(G) \geqq m+1$. If $g$ is exceptional, then, since $G / P_{1}$ satisfies the hypothesis of Theorem $\mathrm{B}, g^{2^{m-1}}$ is in $P_{2} / P_{1}$ if Theorem $\mathrm{B}$ is true. Thus, assuming the validity of $B$, we obtain in all cases $e_{2}\left(G / P_{2}\right) \leqq e_{2}(G)-1$ and Theorem A follows.

3. Proof of Theorem B. Neither the hypothesis nor the conclusion of the theorem is affected by an extension of the field $F$. Hence, without loss of generality, we assume that $F$ is algebraically closed. Since an element of order 2 cannot be exceptional, $m$ must be greater than 1 . Let $\mathrm{h}=g^{2^{2 m-2}}$ and so $h^{2}=g^{2^{m-1}}$.

In proving $B$ we will define subgroups $H$ and $H_{1}$ such that $H \triangleleft G, H_{1} \triangleleft H, h^{2} \in H_{1}$, and $g$ normalizes $H_{1}$. It then will be shown that if $x$ is any element in the largest normal 2-subgroup of $H_{1} / H_{1} \cap N$ then $\left(h^{2}, x\right)=(h, x)^{2}$. From this it will follow that $h^{2}$ is in the largest normal 2-subgroup of $H_{1} / H_{1} \cap N$, and, finally, from this the theorem will follow.

First we need two lemmas which are of use later and which motivate the definition of $H$. Here, and elsewhere, we denote the space on which $G$ operates by $V$.

LEMMA 3.1. If $Q$ is any 2'-subgroup of $G$ which is normalized by $g$, then $h^{2}$ fixes every minimal characteristic $F-Q$ submodule of $V$.

Proof. A minimal characteristic $F-Q$ submodule is simply the join of all those $F-Q$ submodules operator isomorphic to a given irreducible $F-Q$ submodule. Now if $Q$ is a $2^{\prime}$-group, $V$ can be written as the direct sum of the minimal characteristic $F-Q$ submodules. $g$ normalizes $Q$ so $g$ must permute the minimal characteristic $F-Q$ submodules. If the lemma were not true, then $g$, as a permutation of these submodules, would have a cycle of length $2^{m}$ which would contradict the assumption that $g$ is exceptional.

Lemma 3.2. If $Q$ is any abelian 2'-subgroup of $G$ and $x$ is any element of $G$ normalizing $Q$ and fixing every minimal characteristic $F-Q$ submodule of $V$, then $x$ centralizes $Q$.

Proof. Let $V_{i}$ be any minimal characteristic $F-Q$ submodule of $V$. Since $Q$ is abelian and $F$ is algebraically closed, $Q$ operates on $V_{i}$ as a scalar multiplication, i.e., if $y \in Q$ and $v \in V_{i}$ then $y v=\chi_{i}(y) v$ where $\chi_{i}(y)$ is a scalar. We now obtain 


$$
\chi_{i}\left(x^{-1} y x\right) v=x^{-1} y(x v)=x^{-1} \chi_{i}(y) x v=\chi_{i}(y) v .
$$

Thus $(y, x)$ is the identity on $V_{i}$ for all $y \in Q$ and the lemma follows.

Now let $H$ be the normal subgroup of $G$ consisting of all elements which fix every minimal characteristic $F-Q$ submodule for every normal 2 '-subgroup $Q$. Since the largest normal 2-subgroup and the largest normal $2^{\prime}$-subgroup of $H$ are normal in $G$, we see that $H$ has no normal 2-subgroup greater than the identity and the largest normal $2^{\prime}$-subgroup of $H$ is $H \cap N$. By Lemma $3.1 h^{2}$ must belong to $H$.

Let $M$ be the largest normal nilpotent subgroup of $H$. Clearly $M$ is a $2^{\prime}$-group and $M \triangleleft G$. Furthermore, since $H$ is solvable, $M$ contains its own centralizer in $H$ [2].

\section{LEMMA 3.3. $M$ is of class 2.}

Proof. Since $h^{2} \in H, h^{2}$ does not centralize $M$. Thus by Lemmas 3.1 and 3.2, $M$ is not abelian. Now let $c$ be the class of $M$ and suppose $c \geqq 3$. Then if $\Gamma_{i}(M)$ is the $i$ th term in the lower central series of $M\left(\Gamma_{1}(M)=M\right.$ and $\left.\Gamma_{i+1}(M)=\left(\Gamma_{i}(M), M\right)\right)$ and if $d$ is the first integer $\geqq(c+1) / 2$, we have [3, Chap. 10]

$$
\left.\left(\Gamma_{d}(M), M\right)=\Gamma_{a+1}(M) \neq 1 \text { (since } d \leqq c-1\right),
$$

and

$$
\left(\Gamma_{d}(M), \Gamma_{a}(M)\right) \leqq \Gamma_{2 d}(M)=1 .
$$

Thus $\Gamma_{d}(M)$ is abelian and, of course, normal in $G$ but is not centralized by $M$. From Lemma 3.2 and the definition of $H$ we see that this is impossible, and so $c=2$.

$M=M_{1} \times M_{2} \times \ldots$ where $M_{i}$ is the Sylow $q_{i}$-subgroup of $M$ and $q_{i}$ is an odd prime. Each $M_{i}$ is of class at most 2 and so $M_{i}$ is a regular $q_{i}$-group [3, p. 183]. Then the elements of order at most $q_{i}$ form a characteristic subgroup $K_{i}$ of $M_{i}$. Let $K=K_{1} \times K_{2} \times \cdots$ An automorphism of $M_{i}$ of order prime to $q_{i}$ centralizes $K_{i}$ only if it is the identity automorphism [7, Hilfssatz 1.5]. Therefore no 2-element of $H$, except for the identity, centralizes $K$. Hence $K$ cannot be abelian (since $h^{2}$ is a nonidentity 2-element of $H$ ) and so $K$ must be of class 2.

We now are prepared to define the subgroup $H_{1}$. For this purpose decompose $V$ for each $K_{i}$ into the sum

$$
V=V_{i 1} \oplus V_{i 2} \oplus \cdots
$$

where the $V_{i j}$ are the minimal characteristic $F-K_{i}$ submodules. Let 
$C_{i j}=\left\{x \mid x \in H\right.$ and $\left(K_{i}, x\right)=1$ on $\left.V_{i j}\right\} . \mathrm{C}_{i j}$ is a normal subgroup of $H$ although not necessarily normal in $G$.

Take $H_{1}$ to be the intersection of all the $C_{i j}$ which contain $h^{2}$. If $h^{2}$ is not in any $C_{i j}$ then set $H_{1}$ equal to $H$. In any event $H_{1} \triangleleft H$ and $H_{1}$ is normalized by $g$. As was the case with $H, H_{1}$ has no normal 2-subgroup greater than the identity and the greatest normal 2 '-subgroup is $H_{1} \cap N$.

Now let $P$ be a 2-subgroup of $H_{1}$ such that $P$ and $g$ belong to the same Sylow 2-subgroup of $G$ and $P\left(H_{1} \cap N\right) /\left(H_{1} \cap N\right)$ is the largest normal 2-subgroup of $H_{1} /\left(H_{1} \cap N\right)$. Since, modulo $N, P$ is normalized by $g$, it follows that $g$ normalizes $P$.

\section{LEMMA 3.4. If $x \in P$, then $\left(h^{2}, x\right)=(h, x)^{2}$.}

Proof. First we show that this lemma finishes the proof of Theorem B: $h$ normalizes $P$ so that $(h, x)^{2} \in \Phi(P)$ where $\Phi(P)$ is the Frattini subgroup of $P$. Thus the lemma implies that $h^{2}$ centralizes $P / \Phi(P)$. Therefore from [4] we conclude that $h^{2} \in P$. Since $h^{2}$ is in the greatest normal 2-subgroup of $H_{1} /\left(H_{1} \cap N\right)$, it follows that $h^{2}$ is in the greatest normal 2-subgroup of $H /(H \cap N)$ from which the conclusion of Theorem $B$ follows.

To prove the lemma, let $k=\left(h^{2}, x\right)(h, x)^{-2}$ and suppose $k \neq 1$. Since $k$ cannot centralize $K,\left(K_{i}, k\right)$ is not the identity on some $V_{i j}$. Since $k \in H_{1}$, we must have $\left(K_{i}, h^{2}\right)$ also not the identity on $V_{i j}$. (This last statement is the motivation for our choice of $H_{1}$ ).

In what follows let $V^{\prime}=V_{i j}, q=q_{i}$, and $Q, x_{1}, k_{1}$ the restrictions of $K_{i}, x, k$, respectively, to $V^{\prime}$. Let $g^{2^{m-n}}$ be the first power of $g$ fixing $V^{\prime}$ and let $g_{1}$ be the restriction of $g^{2 m-n}$ to $V^{\prime}$. Now $h^{2}$ is not the identity on $V^{\prime}$ and $\left[4\right.$, p. 13] $g_{1}$ must be exceptional

$$
\text { (i.e., } \left.\quad\left(g_{1}-1\right)^{2^{n}-1}=0\right) \text {, }
$$

and thus $n$ must be at least 2. Let $h_{1}=g_{1}^{2 n-2} . \quad k_{1}=\left(h_{1}^{2}, x_{1}\right)\left(h_{1}, x_{1}\right)^{-2}$ and both $\left(Q, h_{1}^{2}\right)$ and $\left(Q, k_{1}\right)$ are not the identity.

Since $g_{1}$ is exceptional and $\left(Q, h_{1}^{2}\right) \neq 1, Q$ cannot be abelian. Thus $Q$ must be of class 2 . $V^{\prime}$ is the sum of absolutely irreducible $F-Q$ submodules all of which are operator isomorphic to each other. Hence $Z(Q)$, the center of $Q$, is cyclic and is generated by a scalar matrix. Since $Q$ is of exponent $q$ and $Q^{\prime} \neq 1$, we see that

$$
Z(Q)=Q^{\prime}=\Phi(Q)
$$

and so $Q$ is an extra-special $q$-group [4, p. 15]. We note also that if $S$ is the 2-group generated by $x_{1}$ and $g_{1}$, then $(Z(Q), S)=1$ since $Z(Q)$ is generated by a scalar matrix. 
Now let $V^{\prime \prime}$ be an irreducible $F-Q S$ submodule of $V^{\prime} . V^{\prime \prime}$ is an irreducible $F-Q$ module [4, Lemma 2.2.3], and $V^{\prime}$ is the sum of $F-Q$ modules operator isomorphic to $V^{\prime \prime}$. Thus $\left(Q, h_{1}^{2}\right) \neq 1$ on $V^{\prime \prime}$ and $g_{1}$ is exceptional on $V^{\prime \prime}$. From [4, Theorem 2.5.4] we have the following:

(1) $2^{n}-1$ is a power of $q$, and

(2) if $g_{1}$ is faithfully and irreducibly represented on $Q_{1} / Q^{\prime}$ (such a $Q_{1}$ can always be found since $h^{2}$ is not the identity on $Q / Q^{\prime}$ ), then $Q$ can be written as the central product of $Q_{1}$ and a group $Q_{2}$ and $g_{1}$ transforms $Q_{2}$ trivially. It now follows [6] that $2^{n}-1=q$ and $\left|Q_{1} / Q^{\prime}\right|=q^{2}$.

The representation of $Q$ on $V^{\prime \prime}$ is isomorphic to the representation of $Q$ on $V^{\prime}$ so that $\left(g_{1}, Q_{2}\right)=1$ on $V^{\prime \prime}$ implies that $\left(g_{1}, Q_{2}\right)=1$. Thus the centralizer of $g_{1}$ in the space $Q / Q^{\prime}$ has co-dimension 2 over $G F(q)$. The minimal equation of $h_{1}$ on $Q_{1} / Q^{\prime}$ must be $t^{2}+1=0$ so that $h_{1}^{2}$ must have the representation

$$
\left(\begin{array}{rr}
-1 & 0 \\
0 & -1
\end{array}\right)
$$

on $Q_{1} / Q^{\prime}$. We now can conclude that for every power of $g_{1}$ (except for the identity, of course), the co-dimension of its centralizer in $Q / Q^{\prime}$ is 2. Also, since $q \equiv 3(\bmod 4), G F(q)$ contains no primitive 4 th root of unity. Thus if $n \neq 2$ then in the completely reduced representation of $g_{1}^{2}$ on $Q / Q^{\prime}$ there is only one nontrivial block. If $n=2$, there are two nontrivial blocks.

Now if $c$ is a generator of $Q^{\prime}$, define $\rho(a, b)$ for $a, b \in Q$ by the equation

$$
(a, b)=c^{\rho(a, b)} .
$$

$\rho(a, b)$ is bilinear and skew symmetric and gives $Q / Q^{\prime}$ the structure of a symplectic space over $G F(q)$ [4].

$\rho$ is of maximum rank since $Q^{\prime}=Z(Q)$ so $Q / Q^{\prime}$ must have dimension $2 r$. Since $\left(S, Q^{\prime}\right)=1, S$ preserves the symplectic structure of $Q / Q^{\prime}$. Thus the representation of $S$ on $Q / Q^{\prime}$ may be considered as a subgroup of a Sylow 2-subgroup of the symplectic group on $Q / Q^{\prime}$.

$Q / Q^{\prime}$ is of dimension $2 r$ over $G F(q)$ so that $Q / Q^{\prime}$ can be provided with the structure of a vector space $U$ of dimension $r$ over $G F\left(q^{2}\right)$. If $u_{1}, \cdots, u_{r}$ is a basis for $U$, the expression [4]

$$
\rho\left(\Sigma \alpha_{i} u_{i}, \Sigma \beta_{i} u_{i}\right)=\Sigma\left(\alpha_{i} \beta_{i}^{\prime}-\alpha_{i}^{\prime} \beta_{i}\right) / \gamma,
$$

where $\alpha^{\prime}=\alpha^{q}$ and $\gamma$ is a primitive 4 th root of unity, is a skew symmetric bilinear form on $U$ of rank $2 r$ with values in $G F(q)$.

Let $\theta$ be a primitive $2^{n+1}$-th root of unity in $G F\left(q^{2}\right)$ and let $T$ be 
the group of transformations of $G F\left(q^{2}\right)$ generated by the two transformations $\alpha \rightarrow \theta^{2} \alpha$ and $\alpha \rightarrow \theta \alpha^{\prime}$. All transformations $\boldsymbol{y}$ of $U$ of the form

$$
\boldsymbol{y}\left(\Sigma \alpha_{i} u_{i}\right)=\Sigma\left(T_{\imath} \alpha_{i}\right) u_{\sigma(\imath)},
$$

where the $T_{i}$ are taken from $T$ and $\sigma$ is a permutation taken from a Sylow 2-subgroup of the symmetric group on the numbers $1,2, \cdots, r$, form a Sylow 2-subgroup of the Symplectic group on $Q / Q^{\prime}$ [4].

Thus we may assume that $\boldsymbol{x}_{1}, \boldsymbol{g}_{1}, \boldsymbol{h}_{1}$, the representations of $x_{1}, g_{1}, h_{1}$, respectively, on $Q / Q^{\prime}$, are of this form. Since $\left(Q, h_{1}^{2}\right) \neq 1$ and $\left(Q, k_{1}\right) \neq 1$, we have $\boldsymbol{h}_{1}^{2} \neq 1$ and $\left(\boldsymbol{h}_{1}^{2}, \boldsymbol{x}_{1}\right) \neq\left(\boldsymbol{h}_{1}, \boldsymbol{x}_{1}\right)^{2}$. We now need more information on $g_{1}$.

Lemma 3.5. The permutation $\sigma$ associated with $\boldsymbol{g}_{1}$ is the identity permutation.

Proof. $\sigma$ is of order less than the order of $g_{1}$ from [4, p. 23]. First suppose $\sigma$ is of order $>2$. Then $n>2$ and so the representation of $g_{1}^{2}$ on $Q / Q^{\prime}$ has only one nontrivial irreducible block. But the permutation associated with $\boldsymbol{g}_{1}^{2}$ is $\sigma^{2}$ which has at least 2 disjoint nontrivial cycles. Clearly this is a contradiction. Thus $\sigma^{2}=1$.

Now suppose $\sigma \neq 1$. Assume, say, $\sigma(1)=2, \sigma(2)=1$. The representation of $g_{1}$ on $Q / Q^{\prime}$ has only one nontrivial irreducble block so $\boldsymbol{g}_{1}$ must be the identity on

$$
\sum_{i \neq 1,2} \alpha_{i} u_{i}
$$

Now $g_{1}^{2}\left(\alpha_{1} u_{1}+\alpha_{2} u_{2}\right)=T_{2} T_{1} \alpha_{1} u_{1}+T_{1} T_{2} \alpha_{2} u_{2}$ and so one of $T_{2} T_{1}$ or $T_{1} T_{2}$ must not be the identity of $T$. But then neither one can be the identity. Therefore the representation of $Q_{/}^{\prime} Q^{\prime}$ would have 2 nontrivial irreducible blocks. This can happen only if $n=2$. This implies that $T_{2} T_{1}$ and $T_{1} T_{2}$ are of order 2 and thus must equal the transformation $\alpha \rightarrow-\alpha$. (This is the only element of order 2 in T.) Thus the centralizer of $g_{1}^{2}$ in $Q / Q^{\prime}$ has co-dimension 4 over $G F(q)$ whereas it should have co-dimension 2. This proves that $\sigma=1$.

Hence $\boldsymbol{g}_{1}$ fixes each $u_{i}$ and must act trivially on $\alpha_{i}$ for all but one value of $i, i=1$, say. Therefore

$$
\boldsymbol{g}_{1}\left(\Sigma \alpha_{i} u_{i}\right)=A \alpha_{1} u_{1}+\sum_{i \neq 1} \alpha_{i} u_{i}
$$

where $A$ is an element of order $2^{n}$ in $T$. Then

$$
\boldsymbol{h}_{1}\left(\Sigma \alpha_{i} u_{i}\right)=A^{2^{n-2}} \alpha_{1} u_{1}+\sum_{i \neq 1} \alpha_{i} u_{i},
$$




$$
\boldsymbol{h}_{1}^{2}\left(\Sigma \alpha_{i} u_{i}\right)=-\alpha_{1} u_{1}+\sum_{i \neq 1} \alpha_{i} u_{i}
$$

We may assume that

$$
\boldsymbol{x}_{1}\left(\Sigma \alpha_{i} u_{i}\right)=\Sigma T_{i} \alpha_{i} u_{\pi(i)}
$$

Case 1. $\pi(1) \neq 1$. Assume, say, that $\pi^{-1}(1)=2$. Straight forward calculation yields

$$
\left(\boldsymbol{h}_{1}, \boldsymbol{x}_{1}\right)\left(\Sigma \alpha_{i} u_{i}\right)=A^{-2^{n-2}} \alpha_{1} u_{1}+T_{2}^{-1} A^{2^{n-2}} T_{2} \alpha_{2} u_{2}+\sum_{i \neq 1,2} \alpha_{i} u_{i} \cdot
$$

But $A^{2^{n-1}}$ is the unique element of order 2 in $T$. Thus

$$
\left(\boldsymbol{h}_{1}, \boldsymbol{x}_{1}\right)^{2}\left(\Sigma \alpha_{i} u_{i}\right)=-\alpha_{1} u_{1}-\alpha_{2} u_{2}+\sum_{i \neq 1,2} \alpha_{i} u_{i}
$$

and it is easily verified that this is the same result as $\left(\boldsymbol{h}_{1}^{2}, \boldsymbol{x}_{1}\right)$.

Case 2: $\pi(1)=1$. In this case we easily find that $\left(\boldsymbol{h}_{1}^{2}, \boldsymbol{x}_{1}\right)$ is the identity while

$$
\left(\boldsymbol{h}_{1}, \boldsymbol{x}_{1}\right)^{2}\left(\Sigma \alpha_{i} u_{i}\right)=\left(A^{2^{n-2}}, T_{1}\right)^{2} \alpha_{1} u_{1}+\sum_{i \neq 1} \alpha_{i} u_{i} .
$$

Now the group $T$ easily is seen to be a generalized quaternion group of order $2^{n+1}$ so that the only conjugates of $A$ in $T$ are $A$ and $A^{-1}$. Thus

$$
\left(A^{2^{n-1}}, T_{1}\right)^{2}=A^{2^{n-1}} T_{1}^{-1}\left(A^{2 n-1}\right) T_{1}=1 .
$$

Thus $\left(\boldsymbol{h}_{1}, \boldsymbol{x}_{1}\right)$ is also the identity.

Therefore it has been shown that

$$
\left(\boldsymbol{h}_{1}, \boldsymbol{x}_{1}\right)^{2}=\left(\boldsymbol{h}_{1}^{2}, \boldsymbol{x}_{1}\right)
$$

in all cases. This completes the proof of lemma 3.4, and, by a previous argument, Theorem B now is proved.

4. Groups with $e_{2}=2$. If $G$ is a solvable group whose Sylow 2 -groups are of exponent 4, then we know from Theorem $\mathrm{A}$ that $l_{2}(G) \leqq 3$. We now investigate conditions for $l_{2}(G) \leqq 2$ to hold. The argument is similar to that used in proving Theorem $B$, but a more restrictive hypothesis is needed. That no loss of generality is involved in assuming the stronger hypothesis is insured by the following reduction theorem, which is stated in a slightly more general form than needed.

A proposition $R$ will be said to be of type 4.1 if it is of the following form:

If $G$ is a finite $p$-solvable group satisfying condition $C$, then 
$l_{p}(G) \leqq f\left(e_{p}(G)\right)$, where $f$ is a monotonically increasing function defined for nonnegative integral arguments, $f(0)=0$, and condition $\mathrm{C}$ either is vacuous or states that $e_{p_{i}}(G) \leqq a_{i}$ for some set, possibly infinite, of primes $p_{i}$ and nonnegative integers $a_{i}$.

Note that the proposition that $l_{2}(G) \leqq e_{2}(G)$ if $G$ is a finite solvable group satisfying $e_{2}(G) \leqq 2$ is of type 4.1. One of the results of this section is that $l_{2}(G) \leqq e_{2}(G)$ if $G$ is a finite group of exponent 12 . This statement is also of type 4.1 since the condition that $G$ be of exponent 12 is equivalent to stating that $e_{2}(G) \leqq 2, e_{3}(G) \leqq 1$, and $e_{p}(G) \leqq 0$ for all other primes.

THEOREM 4.1. To prove a proposition $R$ of type 4.1 it is sufficient to prove the proposition for the following special case:

(1) $G$ is the normal product of $V$ by $G_{1}$ where $V$ is a vector space over $F$, a finite field of characteristic $p$, and $G_{1}$ is a p-solvable linear group on $V$ having no normal p-subgroup other than the identity.

(2) Any irreducible representation of any $p^{\prime}$-subgroup of $G_{1}$ over $F$ is in fact absolutely irreducible.

(3) All groups of order at most $\left|G_{1}\right|$ satisfy $R$.

(4) $V$ is an irreducible $F-G_{1}$ module.

Proof. In proving this theorem we assume $R$ is valid for the special case and then prove it is valid for the general case.

Now suppose $G$ is the group of smallest order which satisfies the hypothesis but not the conclusion of $R$, and let

$$
1=P_{0} \leqq N_{0}<P_{1}<\cdots<P_{l} \leqq N_{l}=G
$$

be the upper $p$-series of $G$. Since $f(0)=0$ we must have $l_{p}(G)>0$. If $F_{1} / N_{0}$ is the Frattini subgroup of $P_{1} / N_{0}$, then, as is shown in [4], $l_{p}\left(G / F_{1}\right)=l_{p}(G)$ so that if $F_{1} \neq 1$ we would have a proper factor group of $G$ satisfying the hypothesis but not the conclusion of $R$.

Hence assume $F_{1}=1$. Thus $P_{1}$ is an elementary abelian $p$-group which we identify with a vector space $V_{1}$ over $G F(p) 。 G / P_{1}$ is faithfully represented as a linear group $G_{1}$ on $V_{1}$ and $G_{1}$ has no normal $p$-group greater than the identity.

From [4, p. 4] we may assume that $G$ has only one minimal normal subgroup. This subgroup must be contained in $V_{1}$ and we denote it with $M$. If $M \neq V_{1}$ and $G_{1}$ is faithfully represented on $V_{1} / M$ then we have $l_{p}(G / M)=l_{p}(G)$ so that we would have a contradiction to the minimality of $G$.

Now suppose $M \neq V_{1}$ and $G_{1}$ is not faithfully represented on $V_{1} / M$. 
Then the elements of $G_{1}$ centralizing $V_{1} / M$ form a normal subgroup of $G_{1}$ greater than the identity. If $Q$ is a minimal normal subgroup of $G_{1}$ centralizing $V_{1} / M$, then $Q$ must be a $p^{\prime}$-group so that $V$ as a $Q$-module is completely reducible. Thus there exists a $Q$-module $M_{1}$ such that $V_{1}=M \oplus M_{1}$. Q is the identity on $M_{1}$ but not on $M$ since $Q$ is faithfully represented on $V_{1}$. Now if $M_{2}$ is the centralizer of $Q$ in $V_{1}$ then $M_{2}$ is normal in $G, M_{2}$ is not the identity, and $M_{2}$ does not contain $M$. This contradicts the minimality of $M$.

Thus we see that $M=V_{1}$ which implies that $G_{1}$ is irreducibly represented on $V_{1}$. A consequence of this is that if $H$ is any normal subgroup greater than the identity in $G_{1}$ then $H$ can have no nonzero fixed vector in $V_{1}$ Otherwise all the vectors fixed by $H$ would form a nontrivial submodule of $V_{1}$.

Now pick $F$ to be a large enough finite extension of $G F(q)$ such that any irreducible representation of any $p^{\prime}$-subgroup of $G_{1}$ over $F$ is absolutely irreducible. Let $1=\theta_{0}, \theta_{1}, \cdots, \theta_{r}$ be a basis for $F$ over $G F(p)$ and let $v_{1}, v_{2}, \cdots, v_{s}$ be a basis for $V_{1}$ over $G F(p)$. Finally let $V$ be the vector space over $F$ with basis $v_{1}, \cdots, v_{s}$, i.e., the vectors of $V$ are the formal sums

$$
\sum_{j=1}^{s} \sum_{i=0}^{r} c_{i j} \theta_{i} v_{j}
$$

where $c_{i j} \in G F(p) . \quad G_{1}$ acts on $V$ in the obvious way.

Consider the group $G^{*}=G_{1} V$, i.e., the normal product of $V$ by $G_{1}$. If $g^{*}$ is of order $p^{m}$ in $G^{*}$ then either the image $g$ of $g^{*}$ in $G_{1}$ is of order $p^{m}$ or $g$ is of order $p^{m-1}$ and $g$ is not exceptional on $V$. In the latter case $(g-1)^{p^{m-1}} v_{i} \neq 0$ for some $v_{i}$ from which it follows that $g$ is not exceptional on $V_{1}$. Thus $e_{p}(G) \geqq(m-1)+1=m$.

Therefore in any event $e_{p}(G) \geqq e_{p}\left(G^{*}\right)$. Since $e_{q}\left(G^{*}\right)=e_{q}(G)$ for $q \neq p, G^{*}$ satisfies condition C. Furthermore $l_{p}(G)=l_{p}\left(G^{*}\right)$ so that if $G^{*}$ satisfies $R$ so does $G$.

Now suppose $H$ is any normal $p^{\prime}$-subgroup other than the identity in $G_{1}$ and suppose

$$
v=\sum_{j=1}^{s} \sum_{i=0}^{r} c_{i j} \theta_{i} v_{j}
$$

is a nonzero vector fixed by $H$. Since $v \neq 0$ the coefficient of $v_{j}$ is not zero for some $j, j=1$ say. Then there exists $\alpha \in F$ such that $\alpha\left(\sum_{i=0}^{r} c_{i 1} \theta_{1}\right)=1$. $H$ must fix $\alpha v$ which can be written in the form $\alpha v=v^{\prime}+v^{\prime \prime}$ where

$$
v^{\prime}=v_{1}+\sum_{j=2}^{s} c_{0 j}^{\prime} v_{j}, v^{\prime \prime}=\sum_{j=2}^{s} \sum_{i=1}^{r} c_{i j}^{\prime} \theta_{i} v_{j} .
$$

For $H$ to fix $\alpha v$ it must also fix $v^{\prime}$ which contradicts the fact that 
$H$ has no nonzero fixed vector in $V_{1}$. Thus $H$ has no nonzero fixed vector in $V$.

If $V$ is an irreducible $F-G_{1}$ module then we have arrived at the special case of the theorem. Therefore assume $U$ is a proper submodule.

If $G_{1}$ is not faithfully represented on $V / U$, then let $Q$ be a minimal normal subgroup of $G_{1}$ centralizing $V / U . Q$ must be a $p^{\prime}$-group so that $V$ is completely reducible as an $F-Q$ module. Thus there exists a nontrivial $F-Q$ submodule on which $Q$ is the identity. This is impossible since $Q$ can have no nonzero fixed vector.

Hence $G_{1}$ is faithfully represented on $V / U$. Thus $l_{p}\left(G^{*}\right)=l_{p}\left(G^{*} / U\right)$ and, of course, $e_{p}\left(G^{*}\right) \geqq e_{p}\left(G^{*} / U\right)$ so that if $G^{*} / U$ satisfies $R$ so does $G^{*}$ and then so does $G$.

We still have that any normal nonidentity $p^{\prime}$-subgroup $H$ of $G_{1}$ has no nonzero fixed vector in $V / U$ since $V$ is completely reducible as an $F-H$ module. Therefore if $G_{1}$ is not irreducibly represented on $V / U$ then the same argument as before yields that $G_{1}$ is faithfully represented on a nontrivial factor module of $V / U$. Continuing in this way we ultimately arrive at the special case where $G_{1}$ is faithfully and irreducibly represented on some vector space over the field $F$. This finishes the proof of Theorem 4.1.

Among the results we now shall prove is that if $G$ is of exponent 12 then $l_{2}(G) \leqq e_{2}(G)$. Before doing this it might be well to justify this work. For in a group of order $2^{a} 3^{b}$ the 2-length and the 3-length can vary at most by one. Thus if it were true that the 3-length of a group of exponent 12 was one, then it would be trivial to state that the 2-length was at most two. However in $[5, \mathrm{p} .5]$ is found an example of a group of exponent 12 but with 3-length two.

For the rest of this paper we make the following standing assumptions.

(1) $G=G_{1} V$, the normal product of $V$ by $G_{1}$, where $V$ is a vector space over a finite field $F$ of characteristic 2 and $G_{1}$ is a finite, solvable linear group having no normal 2-subgroup other than the identity.

(2) $V$ is an irreducible $F-G_{1}$ module.

(3) Any representation over $F$ of any $p^{\prime}$-subgroup of $G_{1}$ is absolutely irreducible.

(4) $e_{2}(G) \leqq 2$.

We are interested in seeing under what conditions can $l_{2}(G)$ exceed $e_{2}(G)$. But if $e_{2}\left(G_{1}\right)=0$ then both $e_{2}(G)$ and $l_{2}(G)$ are 1 , and if $e_{2}\left(G_{1}\right)=1$ then $l_{2}\left(G_{1}\right)=1$ so that $l_{2}(G)=e_{2}(G)=2$. Thus we may as well assume 


\section{(5) $e_{2}\left(G_{1}\right)=2$.}

Later we shall add to these assumptions the further one that $G$ is of exponent 12. Actually, until we restrict ourselves to groups of exponent 12 , we will make no use of the fact that $G_{1}$ is irreducibly represented on $V$.

Now let $N$ be the largest normal 2 '-subgroup of $G_{1}$. We shall show that a certain 2 -subgroup, to be described later, must be contained in the greatest normal 2-subgroup of $G_{1} / N$. In particular if $l_{2}(G)>2$ (which is the same as $l_{2}\left(G_{1}\right)>1$ ), we shall see that there must exist. an element of order 4 of a special type in $G_{1}$.

First let $H$ be the following normal subgroup of $G_{1}: x \in H$ if, and only if, for every normal nilpotent subgroup $Q$ of class at most 2 in $G_{1}, x$ fixes every minimal characteristic $F-Q$ submodule of $V$. A normal nilpotent subgroup of $G_{1}$ must be a $2^{\prime}$-group so that $V$ splits. into the sum of minimal characteristic $F-Q$ modules.

From (5) there are elements of order 4 in $G_{1}$, and from (4) all such elements must be exceptional. Thus if $g$ is of order 4 in $G_{1}$ then $g^{2}$ must be in $H$ by lemma 3.1. Hence $H$ is greater than the identity. $H$ has no normal 2 -subgroup except for the identity and the largest normal 2 '-subgroup is $H \cap N$.

Let $D$ be the greatest normal nilpotent subgroup of $H$. $D=$ $D_{1} \times D_{2} \times \cdots$ where $D_{i}$ is a Sylow $q_{i}$-subgroup of $D$ for an odd prime $q_{i}$. $H$ centralizes any normal abelian subgroup of $G_{1}$ so that, by the proof of Lemma 3.3, we obtain $c(D)=2$. Now, as before, let $K_{i}$ be the subgroup of $D_{i}$ consisting of all elements of order at most $q_{i}$ and let $K=K_{1} \times K_{2} \times \cdots$ We again have that no non-identity 2-element of $H$ centralizes $K$.

Now take $H_{1}$ to be the subgroup of $G_{1}$ consisting of all elements which fix every minimal characteristic $F-K_{i}$ module for all $i . H_{1} \triangleleft G_{1}$, and, since $c\left(K_{i}\right) \leqq 2, H \leqq H_{1}$. $H_{1}$ has no normal 2-subgroup except for the identity and its greatest normal 2 -subgroup is $H_{1} \cap N$.

Let $P$ be a Sylow 2-subgroup of $H_{1} . \quad P \neq 1$ since if $g$ is any element of order 4 in $G_{1}$ then $g^{2} \in H$. Now the square of any element. of $P$ must be in $H$. Thus $P /(P \cap H)$ is of exponent 2 and thus abelian. Therefore $P^{\prime}<H$. We now prove two lemmas which enable us to show directly that $P N / N$ is normal in $G_{1} / N$.

Lemma 4.2. Suppose that $g$ and $h$ are two elements of $P$ and $V^{\prime}$ is a minimal characteristic $F-K_{i}$ submodule of $V$. Let $Q, g_{1}$, and $h_{1}$ be the restrictions of $K_{i}, g$, and $h$, respectively, to $V^{\prime}$. Then if $\left(Q, h_{1}^{2}\right)=1$ it follows that $\left(Q,\left(g_{1}, h_{1}\right)\right)=1$.

Proof. Assume $\left(Q,\left(g_{1}, h_{1}\right)\right) \neq 1$. Therefore neither $g_{1}$ nor $h_{1}$ central- 
izes $Q$. If $\left(Q, g_{1}^{2}\right)=1$, then straight forward calculation yields

$$
\begin{aligned}
& \left(Q,\left(g_{1} h_{1}\right)^{2}\right)=\left(Q,\left(g_{1}, h_{1}\right)\right) \neq 1, \\
& \left(Q,\left(g_{1} h_{1}, h_{1}\right)\right)=\left(Q,\left(g_{1} h_{1}\right)^{-1}\right) \neq 1 .
\end{aligned}
$$

Thus, replacing $g_{1}$ by $g_{1} h_{1}$ if $\left(Q, g_{1}^{2}\right)=1$, we may assume that $\left(Q, g_{1}^{2}\right) \neq 1$ along with $\left(Q, h_{1}^{2}\right)=1$ and $\left(Q,\left(g_{1}, h_{1}\right)\right) \neq 1$.

Now exactly as in the proof of Lemma 3.4 we obtain that $Q$ is an extra special $q$-group (actually $q=3$ since $g_{1}$ is of order 4 and thus exceptional so that $4-1$ must be a power of $q), Q / Q^{\prime}$ is a symplectic space, $g_{1}$ and $h_{1}$ preserve the symplectic structure of $Q / Q^{\prime}$, and we may assume that $g_{1}$ and $h_{1}$ operate on $Q / Q^{\prime}$ as follows:

$$
\begin{aligned}
& \boldsymbol{g}_{1}\left(\Sigma \alpha_{i} u_{i}\right)=A \alpha_{1} u_{1}+\sum_{i \neq 1} \alpha_{i} u_{i}, \\
& \boldsymbol{h}_{1}\left(\Sigma \alpha_{i} u_{i}\right)=\Sigma T_{i} \alpha_{i} u_{\sigma(i)},
\end{aligned}
$$

where $\sigma$ is a permutation of order $\leqq 2$ (since $\left(Q, h_{1}^{2}\right)=1$ ), and $A$ and the $T_{i}$ are chosen from a group isomorphic to the quaternion group of order 8 (since $q=3$ ). In addition $A$ must be of order 4 since $\left(Q, g_{1}^{2}\right) \neq 1$.

If $\sigma$ does not fix 1 then $\left(\boldsymbol{g}_{1}, \boldsymbol{h}_{1}\right)$ would be of order 4 but its centralizer in $Q / Q^{\prime}$ would have co-dimension 4 over $G F(3)$. Thus $\left(\boldsymbol{g}_{1}, \boldsymbol{h}_{1}\right)$ would be of order 4 but not exceptional which is impossible.

Hence $\sigma$ fixes 1 and, since $\left(Q, h_{1}^{2}\right)=1$, we must have

$$
\boldsymbol{h}_{1}\left(\Sigma \alpha_{i} u_{i}\right)= \pm \alpha_{1} n_{1}+\sum_{i \neq 1} T_{i} \alpha_{i} u_{\sigma(i)} .
$$

It is now an easy matter to verify that $\left(\boldsymbol{g}_{1}, \boldsymbol{h}_{1}\right)=1$ and the lemma is proved.

Corollary. If $g, h \in P$ and $h^{2}=1$, then $(g, h)=1$.

Proof. $(g, h)$ is in $P^{\prime}$ and thus in $H$. So if $(g, h) \neq 1$ then $\left(K_{i},(g, h)\right) \neq 1$ for some $K_{i}$. Then lemma states that this cannot happen.

Lemma 4.3. If $g, h \in P$, then $(g, h)^{2}=1$.

Proof. Suppose that $(g, h)^{2} \neq 1$. Then for some $K_{i},\left(K_{i},(g, h)^{2}\right) \neq 1$. Choose $V^{\prime}$ to be a minimal characteristic $F-K_{i}$ submodule of $V$ such that $\left(K_{i},(g, h)^{2}\right)$ is not the identity on $V^{\prime}$. If $Q, g_{1}$, and $h_{1}$ are defined as in the previous lemma, then, if either $\left(Q, g_{1}^{2}\right)$ or $\left(Q, h_{1}^{2}\right)$ is the identity, $\left(g_{1}, h_{1}\right)=1$. Therefore assume neither $g_{1}^{2}$ nor $h_{1}^{2}$ centralize $Q$. Thus $g_{1}$ and $h_{1}$ are both exceptional of order $4 . Q$ is an extra-special 
3-group and we may assume $g_{1}$ and $h_{1}$ operate on $Q / Q^{\prime}$ as follows:

$$
\begin{aligned}
& \boldsymbol{g}_{1}\left(\Sigma \alpha_{i} u_{i}\right)=A \alpha_{1} u_{1}+\sum_{i \neq 1} \alpha_{i} u_{i}, \\
& \boldsymbol{h}_{1}\left(\Sigma \alpha_{i} u_{i}\right)=B \alpha_{j} u_{j}+\sum_{i \neq j} \alpha_{i} u_{i} .
\end{aligned}
$$

Now if $j \neq 1$ then $\left(\boldsymbol{g}_{1}, \boldsymbol{h}_{1}\right)=1$ and if $j=1$ then

$$
\left(\boldsymbol{g}_{1}, \boldsymbol{h}_{1}\right)^{2}\left(\Sigma \alpha_{i} u_{i}\right)=(A, B)^{2} \alpha_{1} u_{1}+\sum_{i \neq 1} \alpha_{i} u_{i} .
$$

But $A$ and $B$ are elements of a quaternion group so that $(A, B)^{2}$ is: the identity and the lemma is proved.

\section{THEOREM 4.4. $P N / N \triangleleft G_{1} / N$.}

Proof. We shall prove that $P\left(H_{1} \cap N\right) /\left(H_{1} \cap N\right) \triangleleft H_{1} /\left(H_{1} \cap N\right)$. which is equivalent to the theorem since $H_{1} \triangleleft G_{1}$.

Let $P_{1}$ be the subgroup of $P$ such that $P_{1}\left(H_{1} \cap N\right) /\left(H_{1} \cap N\right)$ is the largest normal 2-subgroup of $H_{1} /\left(H_{1} \cap N\right) . \quad P_{1} \triangleleft P$ and $P_{1}$ contains the center of $P$ [4, Lemma 1.2.3]. Thus by the corollary to Lemma 4.2, $P_{1}$ contains all elements of order 2 in $P$. The elements of order 2 in $P$ form an elementary abelian group $P_{2}$ which is normal, modulo. $H_{1} \cap N$, in $H_{1}$. The elements of $H_{1} /\left(H_{1} \cap N\right)$ which centralize both $P_{2}$ and $P_{1} / P_{2}$ form a normal subgroup of $H_{1} /\left(H_{1} \cap N\right)$. But if any 2 -element centralized both $P_{2}$ and $P_{1} / P_{2}$, then, as easily may be seen, this element would centralize $P_{1}$ contrary to the fact [4, Lemma 1.2.3] that $P_{1}$ contains its centralizer in $H_{1} /\left(H_{1} \cap N\right)$. Thus the elements. centralizing both $P_{2}$ and $P_{1} / P_{2}$ form a normal 2-subgroup of $H_{1} /\left(H_{1} \cap N\right)$, and from the corollary to Lemma 4.2 and from Lemma 4.3, $P$ must. be contained in this normal 2-subgroup. But $P$ is a Sylow 2-subgroup. of $H_{1}$ and thus it follows that, modulo $H_{1} \cap N, P$ is normal in $H_{1}$.

Corollary. $\quad l_{2}\left(H_{1}\right)=1$.

Now let $S$ be a Sylow 2-subgroup of $G_{1}$ which contains $P$. From. the theorem it follows that $P$ is normal in $S$.

Lemma 4.5. If $P$ contains all elements of order 4 in $S$, then. $l_{2}\left(G_{1}\right)=1$.

Proof. If $S=P$ we are done. Therefore assume $S \neq P$. Then if $x \in S-P$ we must have $x^{2}=1$. Also $x \in S-P, y \in P$ imply that. $x y \in S-P$ so that $(x y)^{2}=1$ which implies that $x^{-1} y x=y^{-1}$. Thus $x$ induces the automorphism $y \rightarrow y^{-1}$ of $P$. This can be an automorphism only if $P$ is abelian. Now if both $x_{1}$ and $x_{2}$ are in $S-P$ then $x_{1} x_{2}$ : 
centralizes $P$. But $e_{2}\left(G_{1}\right)=2$ so that $P$ does contain elements of order 4. Hence $x_{1} x_{2}$ cannot be in $S-P$.

Therefore $|S| P \mid=2$ and $P$ is abelian. Now if $x \in S-P, y \in P$, then $(x, y)=x^{-1} y^{-1} x y=y^{2} \in \Phi(P)$ and thus $x$ centralizes $P / \Phi(P)$. Hence [4, Lemma 1.2.5] $P N / N$ connot be the largest normal 2-subgroup of $G_{1} / N$. But $P$ is maximal in $S$ so that $S N / N$ must be the largest normal 2-subgroup of $G_{1} / N$. This implies that $l_{2}\left(G_{1}\right)=1$.

To our assumptions $(1) \sim(5)$ we now add

\section{(6) $G$ is of exponent 12 .}

This implies that $K$ must be a group of exponent 3 and class at most 2. We prove that $l_{2}\left(G_{1}\right)=1$ in this case by showing that the hypothesis of Lemma 4.5 are satisfied.

For this purpose assume that $g$ is an element of order 4 in $S-P$. $g^{2}$ is in $H$ so $\left(K, g^{2}\right) \neq 1$. Let $V=V_{1} \oplus V_{2} \oplus \cdots$ be the decomposition of $V$ into minimal characteristic $F-K$ modules. Since $g \in S-P, g$ does not fix some $V_{i} \cdot g^{2}$ does fix each $V_{i}$ and if $g^{2}$ is not the identity on a $V_{i}$ then $g$ must fix that $V_{i}$ for otherwise $g$ could not be exceptional [4, p. 13]. We now need the following result:

Lemma 4.6. There exist $x$ and $y$ in $K$ such that $\left(\left(x, g^{2}\right)\right.$, $\left.\left(y, g^{2}\right)\right) \neq 1$.

Proof. Let $C=\left\{x \mid x \in K,\left(x, g^{2}\right) \in Z(K)\right\}$. Clearly $C \geqq Z(K)$ but $C \neq K$ since then $g^{2}$ would centralize $Z(K)$ and $K / Z(K)$ which would imply that $\left(K, g^{2}\right)=1$. $\left(g^{2}\right.$ centralizes $Z(K)$ by Lemma 3.1 and 3.2.) $K / Z(K)$ is an elementary abelian 3-group so that there must be a $G F(3)-g$ module of $K / Z(K)$ complementary to $C / Z(K)$. Thus $K / Z(K)=L / Z(K) \oplus C / Z(K)$ and $g$ normalizes $L$. For all $x \in L-Z(K),\left(x, g^{2}\right)$ is not in $Z(K)$.

Now suppose $x, y \in L-Z(K)$ and $\left(x, g^{2}\right)\left(y, g^{2}\right)^{-1} \in Z(K)$. Since $K / Z(K)$ is abelian, straight forward calculation yields

$$
\begin{aligned}
\left(x y^{-1}, g^{2}\right) & \equiv\left(x, g^{2}\right)\left(y^{-1}, g^{2}\right) & & (\bmod Z(K)), \\
1=\left(y y^{-1}, g^{2}\right) & \equiv\left(y, g^{2}\right)\left(y^{-1}, g^{2}\right) & & (\bmod Z(K)) .
\end{aligned}
$$

Thus $\left(x y^{-1}, g^{2}\right) \equiv\left(x, g^{2}\right)\left(y, g^{2}\right)^{-1} \equiv 1 \quad(\bmod Z(K) . \quad$ This implies that $x y^{-1} \in Z(K)$. Therefore we have shown that $\left(x, g^{2}\right) \equiv\left(y, g^{2}\right)(\bmod Z(K))$ if, and only if, $x \equiv y(\bmod Z(K))$ for $x, y \in L$.

It immediately follows from this that for any $x \in L$, there exists a $y$ such that $x \equiv\left(y, g^{2}\right)(\bmod Z(K))$. Now $L$ cannot be abelian since $g$ normalizes $L$ and $g^{2}$ does not centralize it. From all this we see that there exist $x, y \in L$ such that $\left(\left(x, g^{2}\right),\left(y, g^{2}\right)\right) \neq 1$.

Now taking $x$ and $y$ to satisfy the lemma, we may assume without 
loss of generality that $\left(\left(x, g^{2}\right),\left(y, g^{2}\right)\right)$ is not the identity on $V_{1}$. This implies that $g^{2}$ is not the identity on $V_{1}$ so $g$ must fix $V_{1}$.

Since $g$ does not fix every $V_{i}$, assume $g$ does not fix $V_{2}$. Therefore $g^{2}$ is the identity on $V_{2}$ which then also must be the case for $\left(x, g^{2}\right)$ and $\left(y, g^{2}\right)$.

$V$ is an irreducible $F-G_{1}$ module so that there must be an element taking $V_{1}$ into $V_{2}$. Such an element must be of the form $z h$ where $h \in S$ and $z$ is from a Sylow 3 -subgroup of $G_{1}$ which necessarily must contain $K$. We shall derive a contradiction by showing that $z$ and $K$ generate elements of order 9 which is impossible in a group of exponent 12.

If $h V_{1}=V_{m}$ then $z V_{m}=V_{2}$. Set $g_{1}=h g h^{-1}$. Then

$$
\left(\left(x^{h^{-1}}, g_{1}^{2}\right),\left(y^{h^{-1}}, g_{1}^{2}\right)\right)
$$

is not the identity on $V_{m}$. Now suppose $g_{1} V_{2}=V_{2}$. Then $g h^{-1} V_{2}=$ $h^{-1} V_{2}$, and, since $g V_{2} \neq V_{2}$, this implies that $h^{-1} V_{2}=V_{j}, j \neq 2$. Then we would have $g V_{j}=V_{j}$. But $g h^{-1} \in S$ so that $\left(g h^{-1}\right)^{2} \in H$. Thus $\left(g h^{-1}\right)^{2}$ fixes $V_{2}$ and, therefore, $g h^{-1} V_{j}=V_{2}$. $\left(h^{-1}\right)^{2}$ also must fix $V_{2}$ so we have $h^{-1} V_{j}=V_{2}$. From this we conclude that $V_{2}=g h^{-1} V_{j}=g V_{2}$ which is a contradiction. Hence $g_{1} V_{2} \neq V_{2}$. A consequence of this is that $V_{m} \neq V_{2}$ for $V_{m}=V_{2}$ would imply that $h^{-1} V_{2}=V_{1}$ which would imply that $g_{1} V_{2}=h g V_{1}=V_{2}$. Since $V_{m} \neq V_{2}$ it follows that $z$ is not the identity and so is of order 3 .

If we replace $V_{1}, g, x$, and $y$ by $V_{m}, g_{1}, x^{h^{-1}}$, and $y^{h^{-1}}$, respectively, we may assume that $z V_{1}=V_{2}, g V_{2} \neq V_{2}$, and $\left(\left(x, g^{2}\right),\left(y, g^{2}\right)\right)$ is not the identity on $V_{1}$. Let $x_{1}=\left(x, g^{2}\right)$ and $y_{1}=\left(y, g^{2}\right)$. $x_{1}$ and $y_{1}$ must be the identity on $V_{2}$ since $g_{2}$ is. Since $z$ is of order 3 , we have $z V_{1}=V_{n}$, $z V_{2}=V_{n}(n \neq 1,2)$, and $z V_{n}=V_{1}$.

Let $V^{\prime}=V_{1} \oplus V_{2} \oplus V_{n}$. $V^{\prime}$ is fixed by $z$ and the restrictions of $x_{1}, y_{1}$, and $z$ to $V^{\prime}$ are

$$
z=\left(\begin{array}{ccc}
0 & 0 & A \\
B & 0 & 0 \\
0 & C & 0
\end{array}\right), x_{1}=\left(\begin{array}{rrr}
M & 0 & 0 \\
0 & I & 0 \\
0 & 0 & M_{1}
\end{array}\right), y_{1}=\left(\begin{array}{rrr}
N & 0 & 0 \\
0 & I & 0 \\
0 & 0 & N_{1}
\end{array}\right),
$$

where $I$ is the identity and 0 the zero matrix. Now $\left(x_{1}, y_{1}\right)$ is not the identity on $V_{1}$ but $\left(x_{1}, y_{1}\right) \in Z(K)$ and $Z(K)$ is represented on $V_{1}$ as a cyclic group generated by a scalar matrix. Thus $(M, N)=\omega I$ where $\omega$ is a primitive third root of unity. From $z^{3}=1$ we obtain $C=A^{-1} B^{-1}$.

Now $z, x_{1}$, and $y_{1}$ all belong to the same Sylow 3-subgroup of $G_{1}$. Thus $\left(z x_{1}\right)^{3}=\left(z y_{1}\right)^{3}=1$. From this direct computation yields that $M_{1}=A^{-1} M^{-1} A, N_{1}=A^{-1} N^{-1} A$. Thus $\left(M_{1}, N_{1}\right)=A^{-1}\left(M^{-1}, N^{-1}\right) A$. But $M$ and $N$ generate a group of exponent 3 and class 2 . It follows easily that $\left(M^{-1}, N^{-1}\right)=(M, N)=\omega I$. Thus 


$$
\left(x_{1}, y_{1}\right)=\left(\begin{array}{lrr}
\omega I & 0 & 0 \\
0 & I & 0 \\
0 & 0 & \omega I
\end{array}\right)
$$

It is now a simple matter to verify that $\left(z\left(x_{1}, y_{1}\right)\right)^{3} \neq 1$. Hence $z\left(x_{1}, y_{1}\right)$ is a 3 -element of order greater than 3 which is impossible in a group of exponent 12 . This contradiction proves that the hypothesis of Lemma 4.5 is satisfied and thus:

THEOREM 4.7. If $G$ is a finite group of exponent 12, then $l_{2}(G) \leqq e_{2}(G)$.

\section{REFERENCES}

1. W. Feit and J. Thompson, Solvability of groups of odd order, Pacific J. Math. 13 (1963), 775-1029.

2. H. Fitting, Beitrage zur theorie der gruppen endlichen ordnung, Jber. DMV 48 (1938), 77-141.

3. M. Hall Jr. The Theory of Groups, Macmillan, New York, 1959.

4. P. Hall and H. Higman, On the p-length of $p$-soluble groups and reduction theorems for Burnside's problem, Proc. London Math. Soc. (3) 6 (1956), 1-42.

5. G. Higman, p-length theorems, Proc. of Symposia in Pure Mathematics, AMS 6 (1962), 1-16.

6. A. H. M. Hoare, A Note on 2-soluble groups, J. London Math. Soc. 36 (1960), 193-199.

7. B. Huppert, Subnormale untergruppen und Sylowgruppen, Acta Szeged. 22 (1961), 46-61.

California Institute of Technology

Occidental College

(Now at the University of Alberta, Edmonton) 



\title{
PACIFIC JOURNAL OF MATHEMATICS
}

\author{
EDITORS
}

\author{
H. SAmelson \\ Stanford University \\ Stanford, California \\ R. M. Blumenthal \\ University of Washington \\ Seattle, Washington 98105
}

\author{
J. DugundjI \\ University of Southern California \\ Los Angeles, California 90007
}

*Richard Arens

University of California

Los Angeles, California 90024

\section{ASSOCIATE EDITORS}
E. F. BECKENBACH
B. H. NeUmanN
F. WOLF
K. YoSIDA

\section{SUPPORTING INSTITUTIONS}

\author{
UNIVERSITY OF BRITISH COLUMBIA \\ CALIFORNIA INSTITUTE OF TECHNOLOGY \\ UNIVERSITY OF CALIFORNIA \\ MONTANA STATE UNIVERSITY \\ UNIVERSITY OF NEVADA \\ NEW MEXICO STATE UNIVERSITY \\ OREGON STATE UNIVERSITY \\ UNIVERSITY OF OREGON \\ OSAKA UNIVERSITY \\ UNIVERSITY OF SOUTHERN CALIFORNIA
}

\author{
STANFORD UNIVERSITY \\ UNIVERSITY OF TOKYO \\ UNIVERSITY OF UTAH \\ WASHINGTON STATE UNIVERSITY \\ UNIVERSITY OF WASHINGTON \\ * * * * \\ AMERICAN MATHEMATICAL SOCIETY \\ CALIFORNIA RESEARCH CORPORATION \\ SPACE TECHNOLOGY LABORATORIES \\ NAVAL ORDNANCE TEST STATION
}

Mathematical papers intended for publication in the Pacific Journal of Mathematics should by typewritten (double spaced). The first paragraph or two must be capable of being used separately as a synopsis of the entire paper. It should not contain references to the bibliography. No separate author's resumé is required. Manuscripts may be sent to any one of the four editors. All other communications to the editors should be addressed to the managing editor, Richard Arens, at the University of California, Los Angeles, California 90024.

50 reprints per author of each article are furnished free of charge; additional copies may be obtained at cost in multiples of 50 .

The Pacific Journal of Mathematics is published quarterly, in March, June, September, and December. Effective with Volume 13 the price per volume (4 numbers) is $\$ 18.00$; single issues, $\$ 5.00$. Special price for current issues to individual faculty members of supporting institutions and to individual members of the American Mathematical Society: $\$ 8.00$ per volume; single issues $\$ 2.50$. Back numbers are available.

Subscriptions, orders for back numbers, and changes of address should be sent to Pacific Journal of Mathematics, 103 Highland Boulevard, Berkeley 8, California.

Printed at Kokusai Bunken Insatsusha (International Academic Printing Co., Ltd.), No. 6, 2-chome, Fujimi-cho, Chiyoda-ku, Tokyo, Japan.

PUBLISHED BY PACIFIC JOURNAL OF MATHEMATICS, A NON-PROFIT CORPORATION

The Supporting Institutions listed above contribute to the cost of publication of this Journal, but they are not owners or publishers and have no responsibility for its content or policies.

* Basil Gordon, Acting Managing Editor until February 1, 1966. 


\section{Pacific Journal of Mathematics}

\section{Vol. 15, No. $4 \quad$ December, 1965}

Robert James Blattner, Group extension representations and the structure space ........... 1101

Glen Eugene Bredon, On the continuous image of a singular chain complex .............. 1115

David Hilding Carlson, On real eigenvalues of complex matrices .................... 1119

Hsin Chu, Fixed points in a transformation group ............................. 1131

Howard Benton Curtis, Jr., The uniformizing function for certain simply connected Riemann

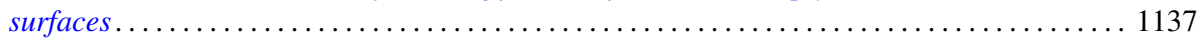

George Wesley Day, Free complete extensions of Boolean algebras................... 1145

Edward George Effros, The Borel space of von Neumann algebras on a separable Hilbert

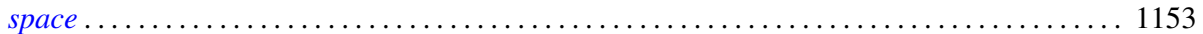

Michel Mendès France, $A$ set of nonnormal numbers ......................... 1165

Jack L. Goldberg, Polynomials orthogonal over a denumerable set ................ 1171

Frederick Paul Greenleaf, Norm decreasing homomorphisms of group algebras . . . . . . . . 1187

Fletcher Gross, The 2-length of a finite solvable group ........................ 1221

Kenneth Myron Hoffman and Arlan Bruce Ramsay, Algebras of bounded sequences ........ 1239

James Patrick Jans, Some aspects of torsion . . . . . . . . . . . . . . . . . . . . . . . 1249

Laura Ketchum Kodama, Boundary measures of analytic differentials and uniform

approximation on a Riemann surface ............................... 1261

Alan G. Konheim and Benjamin Weiss, Functions which operate on characteristic

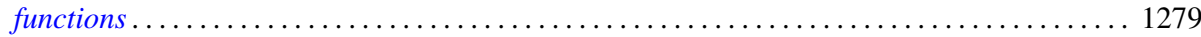

Ronald John Larsen, Almost invariant measures ............................ 1295

You-Feng Lin, Generalized character semigroups: The Schwarz decomposition ............ 1307

Justin Thomas Lloyd, Representations of lattice-ordered groups having a basis . . . . . . . . 1313

Thomas Graham McLaughlin, On relative coimmunity ....................... 1319

Mitsuru Nakai, $\Phi$-bounded harmonic functions and classification of Riemann surfaces ....... 1329

L. G. Novoa, On n-ordered sets and order completeness ..................... 1337

Fredos Papangelou, Some considerations on convergence in abelian lattice-groups . . . . . . . 1347

Frank Albert Raymond, Some remarks on the coefficients used in the theory of homology

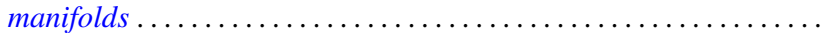

John R. Ringrose, On sub-algebras of a $C^{*}$-algebra .

Jack Max Robertson, Some topological properties of certain spaces of differentiable

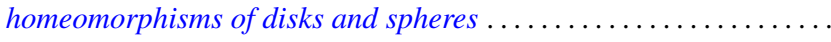

Zalman Rubinstein, Some results in the location of zeros of polynomials

Arthur Argyle Sagle, On simple algebras obtained from homogeneous general Lie triple systems. . . .

Hans Samelson, On small maps of manifolds ............................... 1401

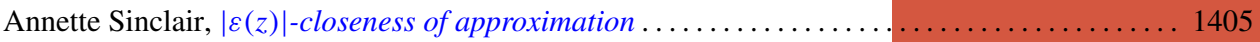

Edsel Ford Stiel, Isometric immersions of manifolds of nonnegative constant sectional curvature

Earl J. Taft, Invariant splitting in Jordan and alternative algebras ................. 1421

L. E. Ward, On a conjecture of R. J. Koch . . . . . . . . . . . . . . . . . . . . . . . . . . . 1429

Neil Marchand Wigley, Development of the mapping function at a corner . . . . . . . . . . 1435

Horace C. Wiser, Embedding a circle of trees in the plane ....................... 1463

Adil Mohamed Yaqub, Ring-logics and residue class rings . . . . . . . . . . . . . . . . 1465

John W. Lamperti and Patrick Colonel Suppes, Correction to: Chains of infinite order and their application to learning theory ........................................ 1471

Charles Vernon Coffman, Correction to: Non-linear differential equations on cones in Banach

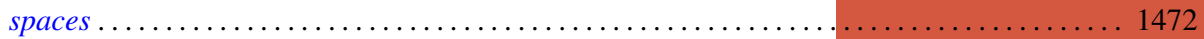

P. H. Doyle, III, Correction to: A sufficient condition that an $\operatorname{arc}$ in $S^{n}$ be cellular. . . . . . . . 1474

P. P. Saworotnow, Correction to: On continuity of multiplication in a complemented algebra 